\title{
Quantifying the Cerebral Hemodynamics of Dural Arteriovenous Fistula in Transverse Sigmoid Sinus Complicated by Sinus Stenosis: A Retrospective Cohort Study
}

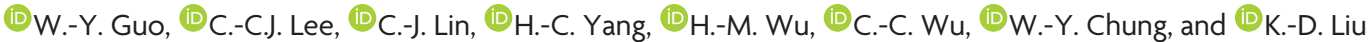

\begin{abstract}
BACKGROUND AND PURPOSE: Sinus stenosis occasionally occurs in dural arteriovenous fistulas. Sinus stenosis impedes venous outflow and aggravates intracranial hypertension by reversing cortical venous drainage. This study aimed to analyze the likelihood of sinus stenosis and its impact on cerebral hemodynamics of various types of dural arteriovenous fistulas.
\end{abstract}

MATERIALS AND METHODS: Forty-three cases of dural arteriovenous fistula in the transverse-sigmoid sinus were reviewed and divided into 3 groups: Cognard type I, type lla, and types with cortical venous drainage. Sinus stenosis and the double peak sign (occurrence of 2 peaks in the time-density curve of the ipsilateral drainage of the internal jugular vein) in dural arteriovenous fistula were evaluated. "TTP" was defined as the time at which a selected angiographic point reached maximum concentration. TTP of the vein of Labbé, TTP of the ipsilateral normal transverse sinus, trans-fistula time, and trans-stenotic time were compared across the 3 groups.

RESULTS: Thirty-six percent of type I, $100 \%$ of type lla, and $84 \%$ of types with cortical venous drainage had sinus stenosis. All sinus stenosis cases demonstrated loss of the double peak sign that occurs in dural arteriovenous fistula. Trans-fistula time (2.09 seconds) and transstenotic time ( 0.67 seconds) in types with cortical venous drainage were the most prolonged, followed by those in type Ila and type I. TTP of the vein of Labbé was significantly shorter in types with cortical venous drainage. Six patients with types with cortical venous drainage underwent venoplasty and stent placement, and 4 were downgraded to type lla.

CONCLUSIONS: Sinus stenosis indicated dysfunction of venous drainage and is more often encountered in dural arteriovenous fistula with more aggressive types. Venoplasty ameliorates cortical venous drainage in dural arteriovenous fistulas and serves as a bridge treatment to stereotactic radiosurgery in most cases.

ABBREVIATIONS: CVD = cortical venous drainage; DAVF = dural arteriovenous fistula; $\mathrm{SRS}=$ stereotactic radiosurgery; $\mathrm{SS}=$ sinus stenosis; TFT = trans-fistula time; $\mathrm{TST}=$ trans-stenotic time; $\mathrm{TTP}_{\mathrm{PV}}=\mathrm{TTP}$ for the parietal vein; $\mathrm{TTP}_{\mathrm{VL}}=\mathrm{TTP}$ of the vein of Labbé

D ural arteriovenous fistulas (DAVFs) account for 10\%-15\% of intracranial vascular malformations. ${ }^{1,2}$ The most common location of an intracranial DAVF is the cavernous sinus, followed by the transverse-sigmoid sinus. ${ }^{1-3}$ Major DAVF classification systems, such as the Cognard and Borden systems, grade DAVFs on the basis of venous drainage patterns, in which the

Received April 1, 2016; accepted after revision August 18.

From the Department of Radiology (W.-Y.G., C.-J.L., H.-M.W., C.-C.W.) and Department of Neurosurgery (C.-C.J.L., H.-C.Y., W.-Y.C., K.-D.L.), Neurological Institute, Taipei Veterans General Hospital, Taipei, Taiwan; and School of Medicine (W.-Y.G., C.-C.J.L., C.-J.L., H.-C.Y., H.-M.W., C.-C.W., W.-Y.C., K.-D.L.), National Yang-Ming University, Taipei, Taiwan.

Dr Chung-Jung Lin is supported by the Taipei Veterans General Hospital (No. V104C-012), and Dr Wan-Yuo Guo receives a collaboration grant from Siemens and Taipei Veterans General Hospital (No. T1100200).

Please address correspondence to Chung-Jung Lin, MD, Department of Radiology, Taipei Veterans General Hospital, No. 201, Section II, Shipai Rd, Taipei, 11217 Taiwan; e-mail: bcjlin@gmail.com; @ChungJungLin

http://dx.doi.org/10.3174/ajnr.A4960 presence of retrograde cortical venous drainage (CVD) indicates a higher risk of hemorrhage. ${ }^{4-7}$ Cases of venous outlet obstruction playing a role in transforming benign (without CVD) into malignant DAVFs (with CVD) have been reported in the literature. ${ }^{8}$ Sinus stenosis (SS) is frequently associated with idiopathic intracranial hypertension. ${ }^{9,10}$ Nevertheless, the incidence of SS and its association with DAVFs have not been thoroughly explored. SS can be found in DAVFs with retrograde or antegrade sinus flow, but its impact on cerebral hemodynamics has rarely been discussed. Theoretically, stenotic and thrombosed sinuses impede the venous outflow, and a DAVF itself increases overall blood volume in the affected sinus; the combination of the 2 hemodynamic disorders adversely affects venous flow and subsequently increases intracranial pressure and the risk of intracranial hemorrhage.

Current treatment strategies for DAVFs in the transverse sinus include microsurgery, endovascular treatment, stereotactic radiosurgery (SRS), or their combinations. ${ }^{1-13}$ Endo- 
vascular treatment has been the treatment of choice for DAVFs with CVD because it provides immediate curative results and minimizes the risk of hemorrhage. ${ }^{14-16}$ Nevertheless, the complication rate of endovascular treatment is higher than that of SRS. ${ }^{14,17,18}$ By contrast, SRS has hardly any periprocedural risks and achieves DAVF cure rates of between $58 \%$ and $73 \%$. Although SRS can reduce the bleeding rate from 20\% to $2 \%$ after shunting has been totally closed, ${ }^{19}$ the latent period for SRS ranges from 1 to 3 years and carries a $4.1 \%$ hemorrhagic rate in DAVFs with CVD. ${ }^{3,20}$ Therefore, SRS is usually preferred for cases without CVD, and endovascular treatment is more suitable for immediately minimizing the risk of hemorrhage.

Several studies have proposed a reconstructive method by using venoplasty and stent placement in combination with transarterial embolization to ameliorate or even cure DAVFs with venous outlet obstruction. ${ }^{21-23}$ We wondered whether this approach could downgrade DAVFs with CVD - that is, to restore their normal cortical venous drainage and make them eligible for SRS, thereby minimizing the risk of hemorrhage during the latent period. Therefore, the purpose of the current study was to clarify the following: 1) the incidence of SS in different grades of DAVF in the transverse sigmoid sinus, 2) the impact of SS on DAVF hemodynamics by using quantitative DSA, and 3) the initial treatment results of venoplasty and/or stent placement followed by SRS.

\section{MATERIALS AND METHODS Patient Population}

The institutional review board of Taipei Veterans General Hospital approved this study. From January 2011 to December 2015, we consecutively recruited cases of angiography-proved DAVFs from the angiosuite logbook. In terms of our inclusion and exclusion criteria, DAVFs involving either side of the transverse-sigmoid sinus were included, while cases already treated before visiting our hospital, DAVFs located elsewhere, and those that did not receive SRS as treatment at all were excluded.

\section{Clinical Presentation, Treatment, and Follow-Up}

Data for symptoms at initial presentation, such as tinnitus, bruit, headaches, previous hemorrhage, and neurologic deficits (ie, visual disturbance, seizure, ataxia, and memory decline), and treatment results during follow-up were based on chart review. There were no missing data for the initial presentation. All patients received SRS and the same follow-up protocol: outpatient department visit and MR imaging at 6-month intervals. Adjunctive endovascular treatment was also recorded. The "primary end point" was complete regression, defined as disappearance of abnormal vasculature on follow-up MR imaging with or without additional angiography. ${ }^{20}$ "Partial regression" was defined as decreased abnormal vascularity compared with the baseline imaging. "Hemorrhage" was defined as any new hemorrhage in the follow-up MR imaging. The "composite end point" (ie, favorable outcome) was defined as complete regression without hemorrhage radiologically. We used the Kaplan-Meier method to handle missing data, treating it as censored. "Restenosis" was defined as a $50 \%$ narrowing of the immediate postvenoplasty sinus diameter during MR imaging follow-up.

\section{Imaging Protocol and Data Analysis}

DSA acquisition with a standard, clinically routine protocol was performed in all 43 cases. A power injector (Liebel-Flarsheim Angiomat; Illumena, San Diego, California) created a contrast bolus by placing a $4 \mathrm{~F}$ angiocatheter in the common carotid artery at the C4 vertebral body level. A bolus of $12-14 \mathrm{~mL}$ of $60 \%$ diluted contrast medium $(340 \mathrm{mg} \mathrm{I} / \mathrm{mL})$ was administered within 1.5 seconds. Neither extra contrast medium nor extra radiation was used. The acquisition parameters were 7.5 frames/s for the first 5 seconds, followed by 4 frames/s for 3 seconds, 3 frames/s for 2 seconds, and finally 2 frames/s for 2 seconds. The entire DSA acquisition process thus normally lasted for 12 seconds, although it was manually prolonged in cases of slow intracranial circulation to allow visualization of internal jugular vein opacification. ${ }^{24}$ All DSAs were performed with the same biplane angiosuite scanner (Axiom Artis; Siemens, Erlangen, Germany). "SS" was defined as the diameter at the stenotic site $<50 \%$ of that of the proximal normal sinus in the lateral view. ${ }^{25}$ All DSA analyses were performed on a workstation equipped with the software syngo iFlow (Siemens). On the basis of the time-density curve, syngo iFlow extracts the time-to-peak of user-selected vascular ROIs on DSA. With the internal carotid artery as a reference, "TTP" was defined as the time point at which the ROI reached the maximum concentration. The "difference in TTP between 2 ROIs" was defined as the time for blood flow to travel between the 2 ROIs; this measure has been validated as a successful surrogate for the pathologic hemodynamics of cerebrovascular disease. ${ }^{24,26-28}$

\section{Definition of Different Time Parameters}

ROIs were placed on the internal carotid artery, ipsilateral transverse sinus, internal jugular vein in the anteroposterior view and the parietal vein, vein of Labbé, and prestenotic and the poststenotic segments of the sinus on lateral views for circulation-time analysis (Fig 1). "Trans-fistula time" (TFT) was defined as the time difference of TTPs between the ICA and internal jugular vein (ie, $\mathrm{TTP}_{\mathrm{JV}}$ ). "Trans-stenotic time" (TST) was defined as the time difference between the TTPs of pre- and poststenotic ROIs. The TTP for the parietal vein $\left(\mathrm{TTP}_{\mathrm{PV}}\right)$ indicated the normal circulation time of normal brain parenchyma. ${ }^{29}$ The TTP for the vein of Labbé $\left(\mathrm{TTP}_{\mathrm{VL}}\right)$ indicated the drainage function of the transverse-sigmoid sinus. The ROI placement was standardized to avoid overlapping anatomic structures and inhomogeneous areas. The caliber of the target vessel was used as the diameter for the ROIs. ${ }^{24}$ The determination of ROIs was performed by a neuroradiologist with 10 years' experience (reader A) and an angiographic technician with 30 years' experience (reader B) who were unaware of the condition of sinus stenosis and clinical features.

\section{Definition of Angiographic Signs}

We modified the method of Riggeal et $\mathrm{al}^{9}$ and defined "venous stenosis" as a diameter $<50 \%$ of the diameter of the normal sigmoid sinus at the stenotic segment. The "double peak sign" refers to the occurrence of 2 peaks in the time-density curve of the ipsi- 


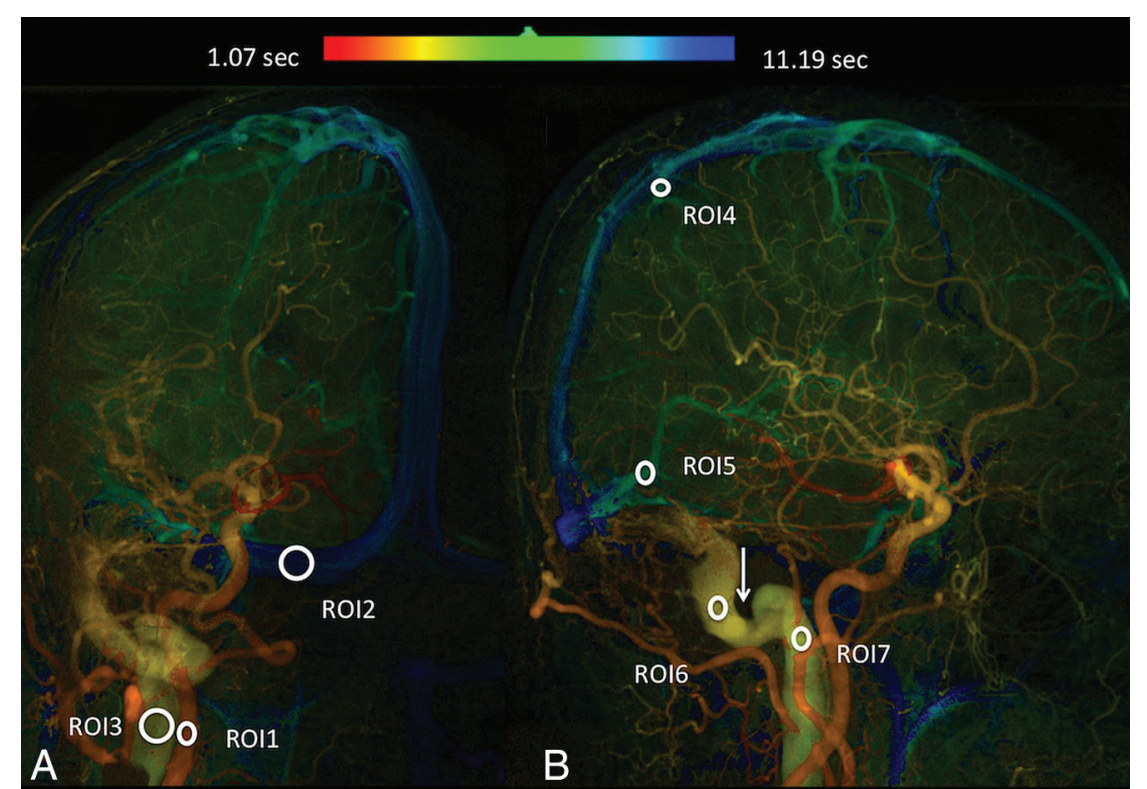

FIG 1. Quantitative color-coded digital subtraction angiography of the anteroposterior $(A)$ and lateral $(B)$ views of a Cognard type I DAVF. ROII: internal carotid artery; ROI2: ipsilateral normal transverse sinus; ROI3: internal jugular vein; ROI4: parietal vein; ROI5: vein of Labbé; ROI6: prestenotic segment; ROI7: poststenotic segment. The Arrow indicates the stenotic sinus segment.

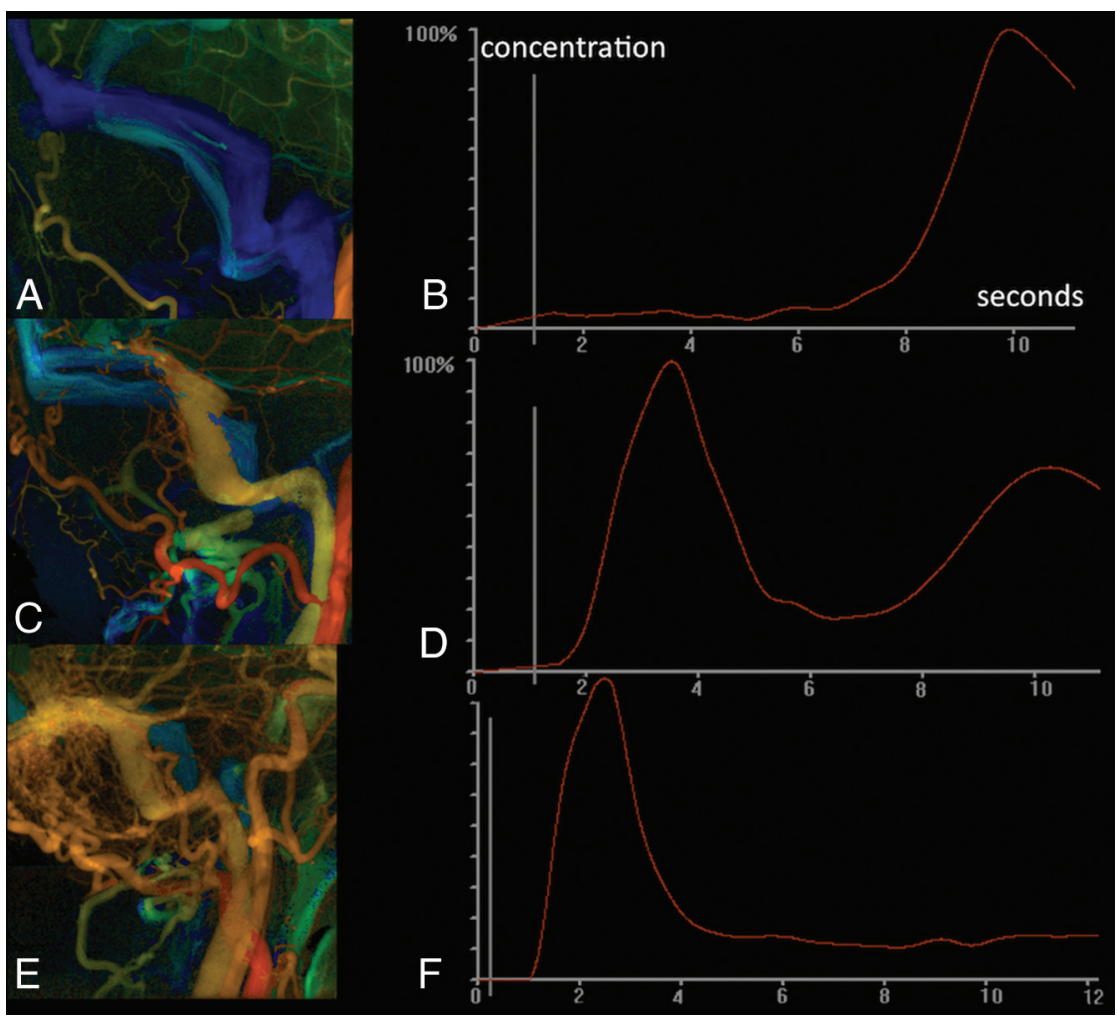

FIG 2. A, Quantitative digital subtraction angiography of a healthy subject. $B$, One single peak appears at 9.87 seconds (venous phase) in the internal jugular vein. C, Quantitative digital subtraction angiography of a Cognard type IDAVF in the left transverse sinus in a 50-year-old woman. $D$, Time-density curve of the internal jugular vein demonstrates 2 peaks. The first peak comes from arterial flow from the DAVF shunt; the second peak comes from blood flow from normal brain parenchyma. E, Quantitative digital subtraction of a case of Cognard type lla $+b$ in the left transverse sigmoid sinus in a 73-year-old man. F, Only a single peak can be depicted in a timedensity curve of the ipsilateral jugular vein, indicating that it lacks the drainage function of the normal brain. lateral drainage internal jugular vein of the DAVF (Fig 2). The first peak indicates shunting arterial blood from the arteriovenous fistula, and the second peak indicates the returning venous blood flows from brain parenchyma. Loss of a double peak with a solitary early peak in cases of DAVF suggests a dysfunction of venous drainage stagnation and may cause venous congestion or venous hypertension. Determinations of sinus stenosis and the double peak sign were made by reader A 1 month after ROI measurement.

\section{Statistical Analysis}

All statistical analyses were performed by using SPSS 20 (2010; IBM, Armonk, New York). The differences in various clinical symptoms, incidences of venous stenosis, and loss of the double peak sign among different DAVF types were compared by using a $\chi^{2}$ test; differences in age and various time parameters among different DAVF types were compared by using an ANOVA test. Inter- and intraobserver variations were evaluated by intraclass classification. The complete regression rate and favorable outcomes were estimated via the KaplanMeier method with a log-rank test. Bonferroni adjustment was applied for post hoc intergroup difference analysis. Significance was set at $P<.018$ for all statistical tests except intraclass classification $(P<.05)$.

\section{RESULTS}

One hundred twenty-six intracranial DAVFs were initially identified from the angiosuite logbook. After excluding 5 patients treated in other hospitals before visiting our hospital, 70 cases of DAVFs in locations other than the transversesigmoid sinus, and 8 patients who had undergone endovascular treatment as the sole treatment, there were 43 DAVFs available for analysis (Fig 3). The cohort consisted of 23 men and 18 women (mean, 56.7 years of age); there were 22 Cognard type I, 8 Cognard type IIa, and 13 Cognard types $\mathrm{IIa}+\mathrm{b}$ or higher DAVFs. Two patients presented with simultaneous cases of bilateral DAVF IIa $+b$ with SS. CVD occurred in all 8 patients who had previous hemorrhage or neurologic deficits other than tinnitus and bruit. No previous hemorrhage 


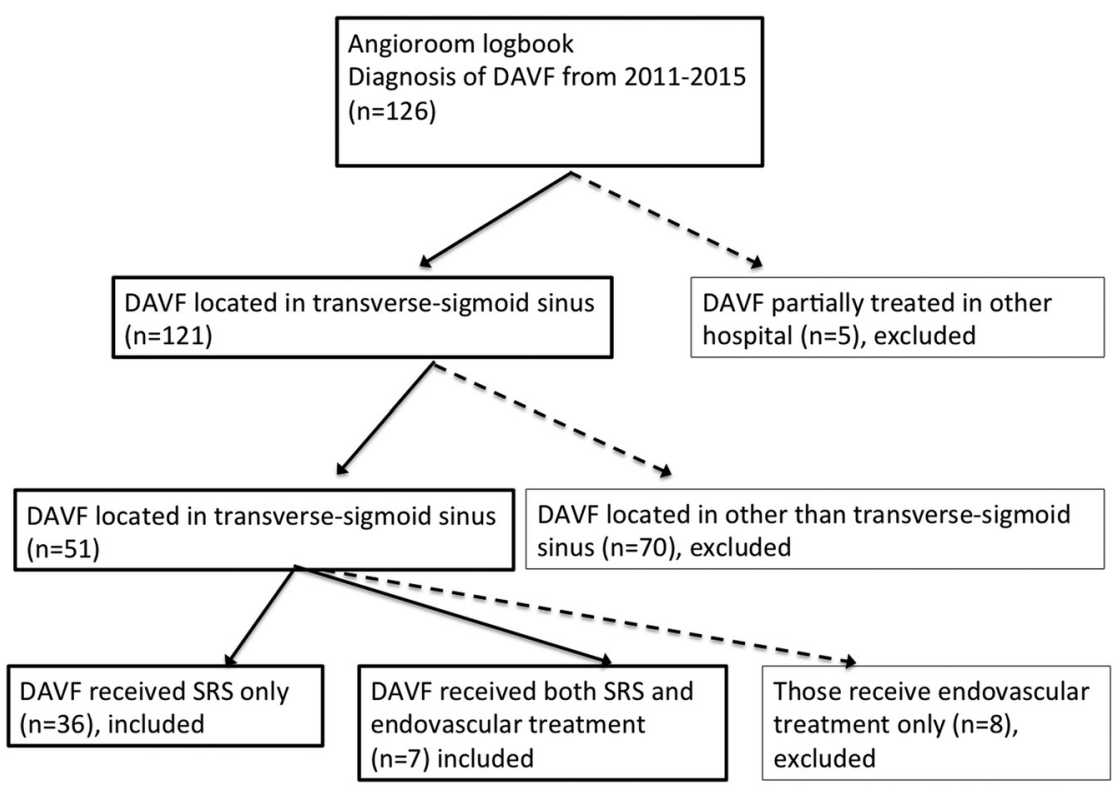

FIG 3. The process of case selection from the angiosuite logbook for our study cohort.

Table 1: Comparison of patient characteristics in 3 different groups: type I, type Ila, and types with CVD

\begin{tabular}{lccc}
\hline & Type I & Type Ila & Types with CVD \\
\hline No. & 22 & 8 & 13 \\
Age $(y r)$ & $58(52.8-64.2)$ & $54(35.5-73.7)$ & $52(41.3-63.8)$ \\
Headaches & $4(18.2 ; 2.1-34.3)$ & $3(37.5 ; 6.2-79.5)$ & $4(30.8 ; 5.7-55.9)$ \\
Hemorrhage/neurologic & 0 & 0 & $8(61.5 ; 35.1-88.0)^{\mathrm{b}}$ \\
$\quad$ deficits & & & \\
Venous stenosis & $8(36.4 ; 16.3-56.5)^{\mathrm{b}}$ & $8(100 \%)$ & $11(84.6 ; 65-100)$ \\
Loss of double peak & $7(31.8 ; 12.4-51.3)^{\mathrm{c}}$ & $8(100 \%)^{\mathrm{c}}$ & $9(69.2 ; 44.1-94.3)^{\mathrm{c}}$ \\
\hline
\end{tabular}

a The numbers inside the parentheses for age indicate the $95 \%$ confidence intervals. The numbers inside the parentheses for headaches, hemorrhage/neurologic deficits, venous stenosis, and loss of double peak indicate the percentage of the observed variable in the group with $95 \%$ confidence intervals.

bignificant difference compared with the other 2 groups.

' Significant difference across the 3 groups.

Table 2: Intra- and interobserver variability in different time parameters $^{\mathrm{a}}$

\begin{tabular}{lccc}
\hline & Reader $\mathbf{A}$ & Reader $\mathbf{B}$ & Interobserver \\
\hline TFT & $0.98(0.96-0.99)$ & $0.97(0.96-0.98)$ & $0.94(0.90-0.97)$ \\
TST & $0.97(0.95-0.99)$ & $0.96(0.94-0.99)$ & $0.93(0.90-0.96)$ \\
TTP $_{\mathrm{PV}}$ & $0.98(0.96-0.99)$ & $0.95(0.90-0.97)$ & $0.91(0.86-0.94)$ \\
TTP $_{\mathrm{VL}}$ & $0.98(0.96-0.99)$ & $0.92(0.89-0.96)$ & $0.92(0.87-0.94)$ \\
TTP $_{\mathrm{TS}}$ & $0.95(0.97-0.91)$ & $0.96(0.93-0.99)$ & $0.94(0.90-0.98)$ \\
\hline
\end{tabular}

Note:- TTP $\mathrm{TS}_{\mathrm{S}}$ indicates TTP of the ipsilateral normal transverse sinus.

a Data are $95 \% \mathrm{Cl}$.

Table 3: Comparison of different time parameters among the 3 groups $^{\mathrm{a}}$

\begin{tabular}{llcc}
\hline & \multicolumn{1}{c}{ Type I } & Type Ila & Types with CVD \\
\hline TFT & $1.04(0.80-1.00)$ & $0.42(0.27-0.4)$ & $2.09(1.06-2.26)^{\mathrm{b}}$ \\
TST & $0.03(0-0)$ & $0.34(0.3-0.53)^{\mathrm{b}}$ & $0.67(-0.54-0.8)^{\mathrm{b}}$ \\
TTP $_{\mathrm{PV}}$ & $4.57(4.14-5.60)$ & $4.25(3.20-4.94)$ & $5.6(4.13-6.26)$ \\
TTP $_{\mathrm{VL}}$ & $4.20(4.26-5.20)$ & $4.40(3.50-6.00)$ & $1.4(0.93-3.46)^{\mathrm{b}}$ \\
TTP $_{\mathrm{TS}}$ & $6.01(4.93-7.47)^{\mathrm{b}}$ & $1.17(1.06-3.6)$ & $1.1(1.06-1.86)$ \\
\hline
\end{tabular}

Note:- TTP $_{\mathrm{TS}}$ indicates TTP of the ipsilateral normal transverse sinus.

a Data are $95 \% \mathrm{Cl}$.

b Significant difference compared with the other 2 groups.

or other neurologic deficits occurred in any of the patients with type I and type IIa. Headaches were observed significantly more often in type IIa and types with CVD (Table 1). Previous hemor- rhage was significantly more frequent in patients with SS. However, neither headaches nor neurologic deficits differed significantly between patients with and without SS.

SS occurred least in Cognard type I ( $n=8,36 \%)$, followed by types with CVD $(n=11,85 \%)$ and type IIa $(n=8$, $100 \%)$. Seven of 8 patients $(88 \%)$ with type I and SS demonstrated a loss of double peaks in their time-density curves. All patients with type IIa and SS had a loss of double peaks. Nine of 13 patients with CVD had a loss of double peaks (Table 1). The other 2 patients with CVD and SS showing double peaks were classified as having types III and IV, respectively, indicating that their venous outlets were still functioning.

The intraclass classification of reader A in different time parameters ranged from 0.95 to 0.98 ; the intraclass classification of reader B ranged from 0.92 to 0.97 . The interobserver variation ranged from 0.91 to 0.94 (Table 2). TFT was significantly prolonged in cases with CVD. Trans-stenotic time differed significantly across the 3 groups: longest in types with CVD (2.09 seconds), followed by type IIa (0.42 seconds), with type I ( 1.04 seconds) having the shortest times. $\mathrm{TTP}_{\mathrm{VL}}$ was significantly reduced in types with CVD (1.4 seconds) compared with type IIa (4.40 seconds) and type I ( 4.20 seconds). There was no significant difference in $\mathrm{TTP}_{\mathrm{PV}}$ among the 3 groups. TTP of the ipsilateral transverse sinus was significantly shortened in type IIa and types with CVD (Table 3).

Six of 13 patients with CVD underwent venoplasty with $(n=$ 4 ) or without $(n=2)$ stent placement before SRS. Peri-stent placement medication consisted of aspirin, $300 \mathrm{mg}$, and clopidogrel, $100 \mathrm{mg}$ daily for 3 days before the procedure and life-long after stent implantation. For those who underwent angioplasty only, the medication was the same as with stent implantation except that the duration of after-procedure medication was shortened to 3 months. Two received post-SRS transarterial embolization due to the presence of new hemorrhage. Only 1 patient received adjunct transarterial embolization before SRS due to existing hemorrhage. Otherwise, transarterial embolization was not performed before SRS in the remaining 12 patients not showing aggressive clinical symptoms and signs. ${ }^{4}$ One type I patient and 2 patients with CVD did not return for follow-up; these 3 patients were not included in subsequent analyses. The average follow-up time of the SRS was $34 \pm 11.5$ months. The complete regression rate was $54.5 \%(12 / 22)$ in type I, 38\% (3/8) in type IIa, and 23\% (3/13) in types with CVD. There was no significant difference in complete regres- 
sion $(P=.176)$ and favorable outcomes $(P=.079)$ among type I, type IIa, type IIa + b, or higher (Fig 4).

All patients experienced improvement of existing pulsatile tinnitus and headaches. Four of the 6 were downgraded to type IIa after combined venoplasty and/or stent placement before SRS treatment. Only 1 patient experienced asymptomatic hemorrhage after the combined treatment, resulting in an annual hemorrhagic rate of $4.0 \%$ after treatment. Follow-up DSA of this patient showed reocclusion of the draining sinus. MR imaging detected 2 cases of restenosis in 4 patients undergoing venoplasty and SRS (Table 4). We kept the 2 patients with restenosis under observation due to their asymptomatic clinical course.

\section{DISCUSSION}

Sinus stenosis is a common associated finding in DAVF in the transverse sigmoid sinus, especially in Cognard types IIa and $\mathrm{II}+\mathrm{b}$. The venous-return from normal parenchyma is predominantly drained via the contralateral normal transverse-sigmoid sinus in all patients with type I with SS. Those patients failed to demonstrate passage of normal brain parenchymal returning blood flow in their ipsilateral jugular veins, which makes them distinct from patients with type I without SS, in which the normal brain parenchyma was still drained via the ipsilateral "healthy" sinus. This finding appears to favor the hypothesis that the stenotic venous outlet plays a role in the progressive development of CVD, pathophysiologically. The hypothesis is supported by Satomi et al, ${ }^{8}$ who reported 2 DAVFs that were longitudinally deteriorated by the development of CVD and venous thrombosis.

In general, the faster the intravascular flow, the shorter the TFT will be. The prolonged TFT in types with CVD in the current study was due to stenotic-induced stagnant flow. In type I, the venous outlet received arterialized antegrade flow and showed no time difference in the peristenotic segment. When the flow reversed in Cognard type II, the TST was prolonged. As SS progressed and CVD developed, the TST was further prolonged (Fig 5). The significantly shorter $\mathrm{TTP}_{\mathrm{VL}}$ in patients with CVD compared with patients with types I and IIa DAVFs without CVD quantitatively reflects the severity of refluxed arterialized venous flow. It could serve as a real-time quantitative regional hemodynamic surrogate marker for treatment strategies used inside the angiosuite. In other words, normalization of $\mathrm{TTP}_{\mathrm{VL}}$ after successful venoplasty and/or stent placement in SS indicates that CVD was caused by sinus outlet obstruction before treatment and was relieved after the interventional procedures.

Several previous reports also described symptomatic relief after venoplasty or stent placement to recanalize the stenotic venous outlets in patients with sinus thrombosis and/or tumor compression. ${ }^{23,30-32}$ The exact etiology of DAVF remains an enigma and might be multifactorial, though most hypotheses hold that it is an acquired disease. ${ }^{33}$ The first hypothesis suggests that DAVF develops from reopening of the existing potential arteriovenous communication due to an increment of sinus pressure. ${ }^{34}$ The second hypothesis asserts that de novo shunts (ie, angiogenesis) develop in response to the stimulation of vascular growth factors in the presence of hypoxia, trauma, or otitis. ${ }^{35,36}$ Venous hypertension induced by an obstruction of the venous

FIG 4. Kaplan-Meier analysis of complete regression ( $A)$ and favorable outcomes $(B)$ among types I, Ila, and Ila + b or higher.

Table 4: Clinical characteristics, treatment strategy, and response in 13 patients with DAVF types with CVD

\begin{tabular}{|c|c|c|c|c|c|c|c|c|}
\hline $\begin{array}{l}\text { Case } \\
\text { No. }\end{array}$ & Sex & $\begin{array}{l}\text { Age } \\
\text { (yr) }\end{array}$ & $\begin{array}{c}\text { Cognard Type } \\
\text { before SRS }\end{array}$ & Treatment & $\begin{array}{c}\text { Cognard Type } \\
\text { after Venoplasty/ } \\
\text { Stent }\end{array}$ & $\begin{array}{l}\text { Treatment } \\
\text { after SRS }\end{array}$ & $\begin{array}{c}\text { Follow-Up } \\
\text { Duration (mo) }\end{array}$ & Response \\
\hline 1 & $\mathrm{~F}$ & 41 & $\mathrm{Il} a+b$ & Venoplasty/stent & Ila & - & 46 & PR \\
\hline 2 & M & 63 & $\mathrm{Ila}+\mathrm{b}$ & Venoplasty & Ila & - & 38 & $\mathrm{CR}$ \\
\hline 3 & $M$ & 56 & $\mathrm{Ila}+\mathrm{b}$ & Venoplasty/stent & Ila & - & 19 & $\mathrm{PR}^{\mathrm{a}}$ \\
\hline 4 & M & 45 & $\mathrm{Ila}+\mathrm{b}$ & - & NA & - & 15 & $\mathrm{CR}$ \\
\hline 5 & $\mathrm{~F}$ & 63 & $\mathrm{Ila}+\mathrm{b}$ & Venoplasty/stent & $\mathrm{Ila}+\mathrm{b}$ & - & 10 & $\mathrm{PR}^{\mathrm{a}}$ \\
\hline 6 & $M$ & 73 & $\mathrm{Ila}+\mathrm{b}$ & - & NA & - & 10 & PR \\
\hline 7 & M & 17 & $\mathrm{Ila}+\mathrm{b}$ & Venoplasty/stent & $\mathrm{Ila}+\mathrm{b}$ & - & 6 & PR \\
\hline 8 & M & 55 & Ilb & - & NA & - & 5.6 & PR \\
\hline 9 & M & 19 & III & - & NA & TAE twice & 17 & PR \\
\hline 10 & M & 27 & III & Venoplasty & Ila & - & 27 & $C R^{b}$ \\
\hline 11 & $M$ & 12 & III & - & & - & 13 & $C R$ \\
\hline 12 & $M$ & 27 & IV & - & & - & 27.6 & PR \\
\hline 13 & $M$ & 55 & IV & - & & - & NA & NA \\
\hline
\end{tabular}

Note:-TAE indicates transarterial embolization; CR, complete regression; PR, partial regression; -, no adjunct treatment was performed; NA, not available.

a Restenosis of sinus after venoplasty and stenting.

${ }^{\mathrm{b}}$ Asymptomatic intracranial hemorrhage on MR imaging. 


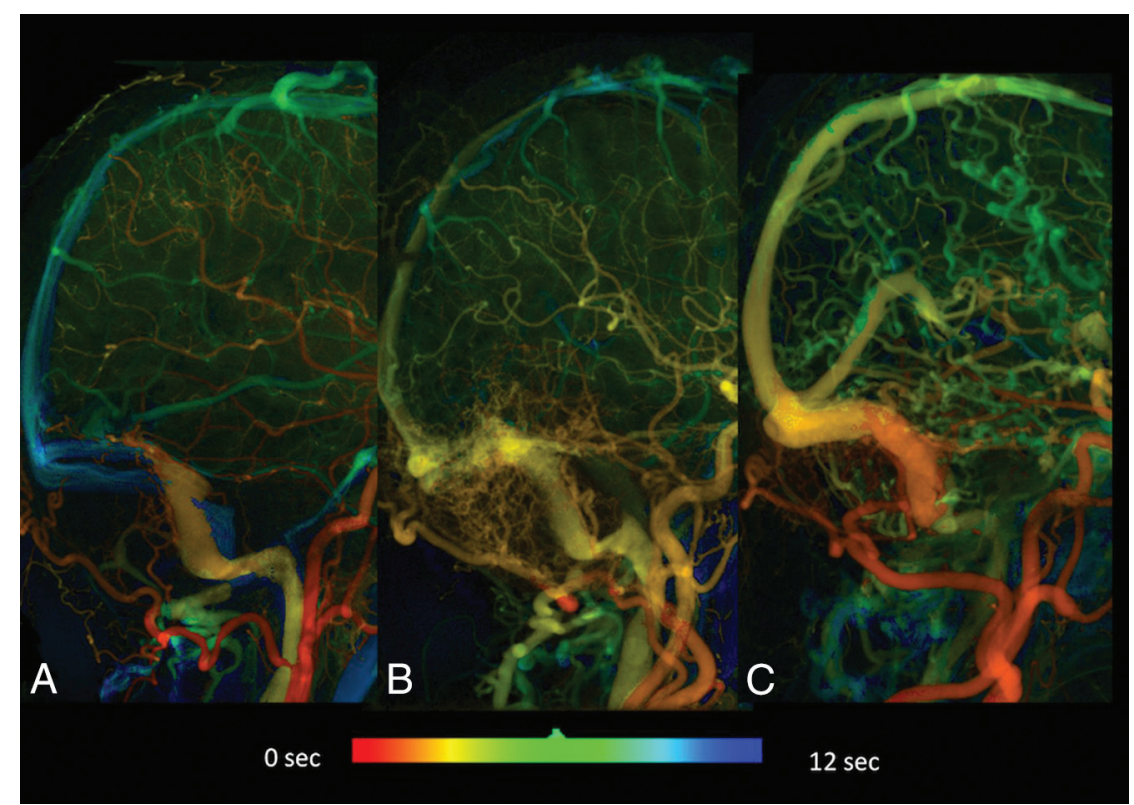

FIG 5. Quantitative DSA of cases of Cognard type I (A), Cognard type lla (B), and Cognard type $\mathrm{Ila}+\mathrm{b}(C)$. Severe sinus stenosis is more commonly encountered in more aggressive DAVF types. The TFT (TTP of the internal jugular vein) was largest in type lla $+b$ (green), followed by types lla (yellow-green) and I (yellow). Progressive shortening of the TTP in the superior sagittal sinuses in type lla and type lla $+b$ is also depicted.

outflow may reduce cerebral perfusion and lead to hypoxia with de novo formation of a DAVF. On the basis of these theories, a correction of the venous hypertension in the sinus should reduce cerebral venous edema and reverse the vicious cycle of creating DAVFs.

None of our patients had a cure of DAVFs by venoplasty and/or stent placement alone. We hypothesize that the application of overlapping stents or stents with a finer network may result in completely blocking the arterial-venous shunts that occur on the sinus wall. ${ }^{21,22}$ Embolization or resection of the sinus is conventionally an option for DAVF types with CVD. The obliteration rate of transarterial embolization in DAVF is $80 \% \cdot{ }^{37}$ If the arterial route to embolize the DAVF also supplies cranial nerves or is technically inaccessible, transvenous embolization might be an option for curing DAVFs. ${ }^{38,39}$ However, sacrifice of a sinus is reserved for cases of an isolated (nonfunctioning) sinus, because sacrificing a functioning sinus might cause deterioration of the CVD and hemorrhage. ${ }^{36}$ We preferred venoplasty and/or stent placement to transarterial embolization followed by SRS because embolizing agents such as Onyx would likely make optimal targeting challenging in both MR imaging and DSA and because tissue ischemia may render the vascular bed less sensitive to radiation and stimulate angiogenesis leading to lesion growth. ${ }^{40,41}$

Nevertheless this multidisciplinary treatment approach will potentially increase the number of unfavorable outcomes in patients before SRS takes effect if a hemorrhage were to occur in the unprotected timeframe. The relative risks of curative embolization attempts alone versus venoplasty and stent placement adjunctive to SRS should be very thoroughly weighed because the latent hemorrhage risk for this combined approach was $4.0 \%$ according to our study. There are several options when facing restenosis: If patients are asymptomatic, they can be kept under obser- vation. If the symptoms persist or are aggravated, then re-stent placement in the sinus, a transarterial approach, or microsurgery can be tried. ${ }^{42}$ In our case, we managed to achieve the benefits of both SRS and endovascular treatment without increasing the periprocedural risks of treating DAVFs with CVD.

There were several limitations to the current study. First, because ours is a tertiary referral medical center for neurologic vascular disorders, the incidences of sinus stenosis might be unusually high due to referring bias. Second, the overall efficacy of combined treatments of venoplasty and/or stent placement followed by SRS warrant a larger scale study with a longer follow-up. Moreover, the aggressiveness of DAVF may also serve as an indicator of response to SRS and warrants individualization of dose selection in SRS. Currently, 2D quantitative DSA merely provides time-based parameters such as time-to-peak to reflect intravascular flow changes. Genuine velocity estimation in DSA relies on 3D acquisitions with an iterative reconstruction algorithm and therefore is not ready for a clinical scenario. ${ }^{43} 3 \mathrm{D}$ quantitative assessment of angiographic morphology and DAVF hemodynamics might further improve the accuracy, whatever treatment strategy is taken. ${ }^{43}$

\section{CONCLUSIONS}

Sinus stenosis is present in nearly one-third of cases of Cognard type I DAVF in the transverse-sigmoid sinus and is more frequently encountered in patients with more aggressive types. Loss of the double peak time-density curve of ipsilateral sinus flow in a DAVF suggests dysfunction of venous drainage and warrants urgent treatment. Venoplasty and/or stent placement of the dysfunctional sinus may downgrade the DAVF and make it amenable to SRS with less risk of hemorrhage in the latent period in most cases.

\section{ACKNOWLEDGMENTS}

We thank Chung-Hsien Lin for his assistance with the statistical analysis.

Disclosures: Wan-Yuo Guo-RELATED: Grant: Siemens, Comments: This work is supported in part by a collaboration contract between Taipei Veterans General Hospital and Siemens. * Chung-Jung Lin—RELATED: Grant: Taipei Veterans General Hospital (No. V104-C-012). * ${ }^{*}$ Money paid to the institution.

\section{REFERENCES}

1. Piippo A, Niemelä M, van Popta J, et al. Characteristics and longterm outcome of 251 patients with dural arteriovenous fistulas in a defined population. J Neurosurg 2013;118:923-34 CrossRef Medline

2. Kim MS, Han DH, Kwon O-K, et al. Clinical characteristics of dural arteriovenous fistula. J Clin Neurosci 2002;9:147-55 CrossRef Medline

3. Chen CJ, Lee CC, Ding D, et al. Stereotactic radiosurgery for intracranial dural arteriovenous fistulas: a systematic review. J Neurosurg 2015;122:353-62 CrossRef Medline 
4. Söderman M, Pavic L, Edner G, et al. Natural history of dural arteriovenous shunts. Stroke 2008;39:1735-39 CrossRef Medline

5. Davies MA, Ter Brugge K, Willinsky R, et al. The natural history and management of intracranial dural arteriovenous fistulae: part 2, aggressive lesions. Interv Neuroradiol 1997;3:303-11 Medline

6. van Rooij WJ, Sluzewski M, Beute GN. Dural arteriovenous fistulas with cortical venous drainage: incidence, clinical presentation, and treatment. AJNR Am J Neuroradiol 2007;28:651-55 Medline

7. van Dijk JM, terBrugge KG, Willinsky RA, et al. Clinical course of cranial dural arteriovenous fistulas with long-term persistent cortical venous reflux. Stroke 2002;33:1233-36 CrossRef Medline

8. Satomi J, van Dijk JM, Terbrugge KG, et al. Benign cranial dural arteriovenous fistulas: outcome of conservative management based on the natural history of the lesion. J Neurosurg 2002;97:767-70 CrossRef Medline

9. Riggeal BD, Bruce BB, Saindane AM, et al. Clinical course of idiopathic intracranial hypertension with transverse sinus stenosis. Neurology 2013;80:289-95 CrossRef Medline

10. Degnan AJ, Levy LM. Pseudotumor cerebri: brief review of clinical syndrome and imaging findings. AJNR Am J Neuroradiol 2011;32: 1986-93 CrossRef Medline

11. Vanlandingham M, Fox B, Hoit D, et al. Endovascular treatment of intracranial dural arteriovenous fistulas. Neurosurgery 2014; 74(suppl 1):S42-49 CrossRef Medline

12. Söderman M, Dodoo E, Karlsson B. Dural arteriovenous fistulas and the role of gamma knife stereotactic radiosurgery: the Stockholm experience. Prog Neurol Surg 2013;27:205-17 CrossRef Medline

13. Yang $\mathrm{H}$, Kano $\mathrm{H}$, Kondziolka $\mathrm{D}$, et al. Stereotactic radiosurgery with or without embolization for intracranial dural arteriovenous fistulas. Prog Neurol Surg 2013;27:195-204 CrossRef Medline

14. van Rooij WJ, Sluzewski M. Curative embolization with Onyx of dural arteriovenous fistulas with cortical venous drainage. AJNR Am J Neuroradiol 2010;31:1516-20 CrossRef Medline

15. Stiefel MF, Albuquerque FC, Park MS, et al. Endovascular treatment of intracranial dural arteriovenous fistulae using Onyx: a case series. Neurosurgery 2009;65:132-39; discussion 139-40 Medline

16. Macdonald JH, Millar JS, Barker CS. Endovascular treatment of cranial dural arteriovenous fistulae: a single-centre, 14-year experience and the impact of Onyx on local practise. Neuroradiology 2010; 52:387-95 CrossRef Medline

17. Yoshida K, Melake M, Oishi H, et al. Transvenous embolization of dural carotid cavernous fistulas: a series of $\mathbf{4 4}$ consecutive patients. AJNR Am J Neuroradiol 2010;31:651-55 CrossRef Medline

18. Zenteno M, Santos-Franco J, Rodríguez-Parra V, et al. Management of direct carotid-cavernous sinus fistulas with the use of ethylenevinyl alcohol (Onyx) only: preliminary results. J Neurosurg 2010; 112:595-602 CrossRef Medline

19. Plasencia AR, Santillan A. Embolization and radiosurgery for arteriovenous malformations. Surg Neurol Int 2012;3(suppl 2):S90S104 CrossRef Medline

20. Pan DH, Chung WY, Guo WY, et al. Stereotactic radiosurgery for the treatment of dural arteriovenous fistulas involving the transverse-sigmoid sinus. J Neurosurg 2002;96:823-29 CrossRef Medline

21. Liebig $\mathrm{T}, \mathrm{Henkes} \mathrm{H}$, Brew $\mathrm{S}$, et al. Reconstructive treatment of dural arteriovenous fistulas of the transverse and sigmoid sinus: transvenous angioplasty and stent deployment. Neuroradiology 2005;47: 543-51 CrossRef Medline

22. Levrier O, Métellus P, Fuentes S, et al. Use of a self-expanding stent with balloon angioplasty in the treatment of dural arteriovenous fistulas involving the transverse and/or sigmoid sinus: functional and neuroimaging-based outcome in 10 patients. J Neurosurg 2006; 104:254-63 CrossRef Medline

23. Xu K, Yu T, Yuan Y, et al. Current status of the application of intracranial venous sinus stenting. Int J Med Sci 2015;12:780-89 CrossRef Medline

24. Lin CJ, Hung SC, Guo WY, et al. Monitoring peri-therapeutic cerebral circulation time: a feasibility study using color-coded quanti- tative DSA in patients with steno-occlusive arterial disease. AJNR Am J Neuroradiol 2012;33:1685-90 CrossRef Medline

25. Lin CJ, Chang FC, Tsai FY, et al. Stenotic transverse sinus predisposes to poststenting hyperperfusion syndrome as evidenced by quantitative analysis of peritherapeutic cerebral circulation time. AJNR Am J Neuroradiol 2014;35:1132-36 CrossRef Medline

26. Levitt MR, Morton RP, Haynor DR, et al. Angiographic perfusion imaging: real-time assessment of endovascular treatment for cerebral vasospasm. J Neuroimaging 2014;24:387-92 CrossRef Medline

27. Gölitz P, Struffert T, Lücking H, et al. Parametric color coding of digital subtraction angiography in the evaluation of carotid cavernous fistulas. Clin Neuroradiol 2013;23:113-20 CrossRef Medline

28. Strother CM, Bender F, Deuerling-Zheng Y, et al. Parametric color coding of digital subtraction angiography. AJNR Am J Neuroradiol 2010;31:919-24 CrossRef Medline

29. Greitz T. A radiologic study of the brain circulation by rapid serial angiography of the carotid artery. Acta Radiol Suppl 1956;140:1-123 Medline

30. Tsumoto T, Miyamoto T, Shimizu M, et al. Restenosis of the sigmoid sinus after stenting for treatment of intracranial venous hypertension: case report. Neuroradiology 2003;45:911-15 CrossRef Medline

31. Ganesan D, Higgins JN, Harrower T, et al. Stent placement for management of a small parasagittal meningioma: technical note. J Neurosurg 2008;108:377-81 CrossRef Medline

32. Hirata E, Higashi T, Iwamuro Y, et al. Angioplasty and stent deployment in acute sinus thrombosis following endovascular treatment of dural arteriovenous fistulae. J Clin Neurosci 2009;16:725-27 CrossRef Medline

33. Gupta A, Periakaruppan A. Intracranial dural arteriovenous fistulas: a review. Indian J Radiol Imaging 2009;19:43-48 CrossRef Medline

34. Houser OW, Campbell JK, Campbell RJ, et al. Arteriovenous malformation affecting the transverse dural venous sinus: an acquired lesion. Mayo Clin Proc 1979;54:651-61 Medline

35. Tirakotai W, Bertalanffy H, Liu-Guan B, et al. Immunohistochemical study in dural arteriovenous fistulas and possible role of local hypoxia for the de novo formation of dural arteriovenous fistulas. Clin Neurol Neurosurg 2005;107:455-60 CrossRef Medline

36. Lasjaunias P, BrensteinA, ter Brugge KG. Surgical Neuroangiography. Berlin: Springer; 2004:565-607

37. Cognard C, Januel AC, Silva NA Jr, et al. Endovascular treatment of intracranial dural arteriovenous fistulas with cortical venous drainage: new management using Onyx. AJNR Am J Neuroradiol 2008;29:235-41 CrossRef Medline

38. Natarajan SK, Ghodke B, Kim LJ, et al. Multimodality treatment of intracranial dural arteriovenous fistulas in the Onyx era: a single center experience. World Neurosurg 2010;73:365-79 CrossRef Medline

39. Lekkhong E, Pongpech S, Ter Brugge K, et al. Transvenous embolization of intracranial dural arteriovenous shunts through occluded venous segments: experience in $\mathbf{5 1}$ patients. AJNR Am J Neuroradiol 2011;32:1738-44 CrossRef Medline

40. Akakin A, Ozkan A, Akgun E, et al. Endovascular treatment increases but gamma knife radiosurgery decreases angiogenic activity of arteriovenous malformations: an in vivo experimental study using a rat cornea model. Neurosurgery 2010;66:121-29; discussion 129-30 CrossRef Medline

41. Mullan S, Mojtahedi S, Johnson DL, et al. Embryological basis of some aspects of cerebral vascular fistulas and malformations. J Neurosurg 1996;85:1-8 CrossRef Medline

42. Choi BJ, Lee TH, Kim CW, et al. Reconstructive treatment using a stent graft for a dural arteriovenous fistula of the transverse sinus in the case of hypoplasia of the contralateral venous sinuses: technical case report. Neurosurgery 2009;65:E994-96; discussion E996 CrossRef Medline

43. Chen GH, Li Y. Synchronized multiartifact reduction with tomographic reconstruction (SMART-RECON): a statistical model based iterative image reconstruction method to eliminate limitedview artifacts and to mitigate the temporal-average artifacts in time-resolved CT. Med Phys 2015;42:4698-707 CrossRef Medline 